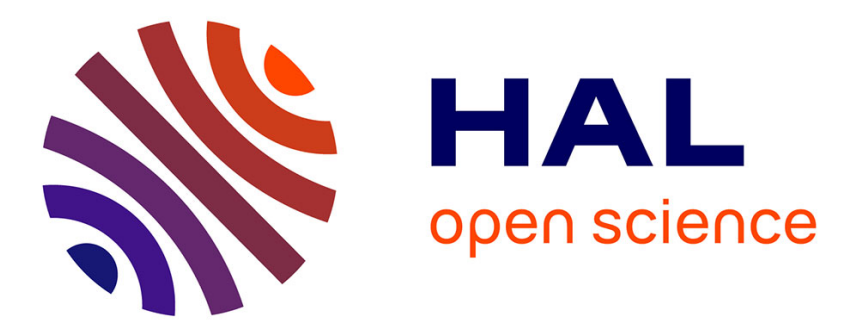

\title{
Neurite density is reduced in the presymptomatic phase of C9orf72 disease
}

Junhao Wen, Hui Zhang, Daniel C Alexander, Stanley Durrleman, Alexandre Routier, Daisy Rinaldi, Marion Houot, Philippe Couratier, Didier Hannequin, Florence Pasquier, et al.

\section{- To cite this version:}

Junhao Wen, Hui Zhang, Daniel C Alexander, Stanley Durrleman, Alexandre Routier, et al.. Neurite density is reduced in the presymptomatic phase of C9orf72 disease. Journal of Neurology, Neurosurgery and Psychiatry, 2019, J Neurol Neurosurg Psychiatry, 90 (4), pp.387-94. 10.1136/jnnp-2018-318994 . hal-01907482

\section{HAL Id: hal-01907482 \\ https://hal.inria.fr/hal-01907482}

Submitted on 29 Oct 2018

HAL is a multi-disciplinary open access archive for the deposit and dissemination of scientific research documents, whether they are published or not. The documents may come from teaching and research institutions in France or abroad, or from public or private research centers.
L'archive ouverte pluridisciplinaire HAL, est destinée au dépôt et à la diffusion de documents scientifiques de niveau recherche, publiés ou non, émanant des établissements d'enseignement et de recherche français ou étrangers, des laboratoires publics ou privés. 


\section{Neurite density is reduced in the presymptomatic phase of}

\section{C9orf72 disease}

Junhao Wen, $\mathrm{MS}^{1,2}$, Hui Zhang, $\mathrm{PhD}^{3}$, Daniel C. Alexander, $\mathrm{PhD}^{3}$, Stanley Durrleman, $\mathrm{PhD}$ 1,2, Alexandre Routier, $\mathrm{MS}^{1,4}$, Daisy Rinaldi, $\mathrm{PhD}^{5,6}$, Marion Houot, $\mathrm{MS}^{7}$, Philippe Couratier, MD, $\mathrm{PhD}^{8,9}$, Didier Hannequin, $\mathrm{MD}, \mathrm{PhD}^{10,11}$, Florence Pasquier, $\mathrm{MD}, \mathrm{PhD}^{12,13}$, Jiaying Zhang, $\mathrm{PhD}^{3}$, Olivier Colliot, $\mathrm{PhD}^{1,6,14}$, Isabelle Le Ber, $\mathrm{MD}, \mathrm{PhD}^{5,6,15}$, Anne Bertrand, MD, $\mathrm{PhD}^{1,6,16, *}$;

for the Predict to Prevent Frontotemporal Lobar Degeneration and Amyotrophic Lateral Sclerosis (PREV-DEMALS) Study Group

*Deceased, March $2^{\text {nd }} 2018$

${ }^{1}$ Inria Paris, Aramis project-team, Paris, France

${ }^{2}$ Sorbonne Université, Inserm, CNRS, Institut du Cerveau et la Moelle (ICM), Paris, France

${ }^{3}$ Department of Computer Science and Centre for Medical Image Computing, University College London, London, United Kingdom

${ }^{4}$ Sorbonne Université, Inserm, CNRS, Institut du Cerveau et la Moelle (ICM), FrontLab, Paris, France

${ }^{5}$ AP-HP, Hôpital Pitié-Salpêtrière, Centre de Référence des Démences Rares ou Précoces, Paris, France

${ }^{6}$ Sorbonne Université, Inserm, CNRS, Institut du Cerveau et la Moelle (ICM), AP-HP, Paris, France

${ }^{7}$ AP-HP, Hôpital Pitié-Salpêtrière, Institute of Memory and Alzheimer's Disease (IM2A), Centre of excellence of neurodegenerative disease (CoEN), Department of Neurology, ICM, CIC Neurosciences, Paris, France 
${ }^{8}$ Department of Neurology, Centre de Compétences Démences Rares, Centre Hospitalier Universitaire de Limoges, Limoges, France

${ }^{9}$ Limoges University, UMR1094, Limoges, France

${ }^{10}$ Centre National de Référence pour les Malades Alzheimer Jeunes, Centre Hospitalier Universitaire de Rouen, INSERM 1245, Rouen, France

${ }^{11}$ Department of Neurology, Centre Hospitalier Universitaire de Rouen, Rouen, France

${ }^{12}$ Centre National de Référence pour les Malades Alzheimer Jeunes, Centre Hospitalier Universitaire de Lille, France

${ }^{13}$ Université de Lille, INSERM U1171, Labex DistALZ, CoEN LiCEND, Lille, France ${ }^{14}$ AP-HP, Departments of Neuroradiology and Neurology, Pitié-Salpêtrière Hospital, Paris, France

${ }^{15}$ AP-HP, Hôpital Pitié-Salpêtrière, Institute of Memory and Alzheimer's Disease (IM2A), Centre of excellence of neurodegenerative disease $(\mathrm{CoEN})$, Department of Neurology, Paris, France

${ }^{16}$ AP-HP, Saint-Antoine Hospital, Department of Radiology, Paris, France

\section{Corresponding author:}

Isabelle Le Ber

Institut du Cerveau et la Moelle (ICM) ; Pitié-Salpêtrière Hospital ; 47-83, boulevard de l’Hôpital, 75651 Paris Cedex 13, France. Telephone: 003315727 4682; Fax: 003315727 4795. E-mail: isabelle.leber@upmc.fr 


\section{Abstract}

OBJECTIVE. To assess the added value of neurite orientation dispersion and density imaging (NODDI) compared to conventional DTI and anatomical MRI to detect changes in presymptomatic carriers of chromosome 9 open reading frame 72 (C9orf72) mutation.

METHODS. The PREV-DEMALS study is a prospective, multicenter, observational study of first-degree relatives of individuals carrying the $C 9$ orf 72 mutation. Sixty-seven participants (38 presymptomatic C9orf72 mutation carriers [C9+], 29 non carriers [C9-]) were included in the present cross-sectional study. Each participant underwent one single-shell, multi-shell diffusion MRI and 3DT1 MRI. Volumetric measures, DTI and NODDI metrics were calculated within regions of interest. Differences in white matter integrity, gray matter volume and free water fraction between $C 9+$ and $C 9$ - individuals were assessed using linear mixed-effects models.

RESULTS. Compared with $C 9-, C 9+$ demonstrated white matter abnormalities in 10 tracts with neurite density index, and only 5 tracts with DTI metrics. Effect size was significantly higher for the neurite density index than for DTI metrics in two tracts. No tract had a significantly higher effect size for DTI than for NODDI. For gray matter cortical analysis, free water fraction was increased in 13 regions in $\mathrm{C} 9+$, whereas 11 regions displayed volumetric atrophy.

CONCLUSIONS. NODDI provides higher sensitivity and greater tissue-specificity compared to conventional DTI for identifying white matter abnormalities in the presymptomatic C9orf72 carriers. Our results encourage the use of neurite density as biomarker of the preclinical phase. Clinical trial identifier number NCT02590276 on http://clinicaltrials.gov/. 


\section{Introduction}

Frontotemporal lobar degeneration (FTLD) and amyotrophic lateral sclerosis (ALS) are two degenerative diseases that share common genetic causes, the most frequent being a GGGGCC repeat expansion in the chromosome 9 open reading frame 72 (C9orf72) gene. ${ }^{1,2}$ Early stages of $C 9$ orf 72 carriers have received much interest, because presymptomatic carriers represent the optimal target population for the development of new disease-modifying treatments against FTLD and ALS. Anatomical magnetic resonance imaging (MRI) derived metrics, such as volumetry, have revealed brain atrophy in presymptomatic individuals who carry the $C 9$ orf 72 mutation $(C 9+)^{3,4,5,6,7,8,9}$ Three studies ${ }^{5,6,8}$ also detected disruptions of white matter integrity using diffusion tensor imaging (DTI) technique, whereas another study failed to identify such abnormalities. ${ }^{4}$ The DTI results are promising but with limitations. First, DTI metrics, such as fractional anisotropy (FA), are non-specific biomarker of microstructural architecture. ${ }^{10,11}$ For instance, FA changes could be underpinned by combinations of neurite density reduction and orientation dispersion changes. ${ }^{12}$ Besides, DTI is limited when an image voxel suffers from partial volume effect. Neurite orientation dispersion and density imaging (NODDI) was proposed to characterize alterations of microstructural integrity with higher tissue-specificity. ${ }^{12}$ NODDI derives a neurite density index (NDI) and orientation dispersion index (ODI) to quantify the density and angular variation of neurites, respectively. In addition, NODDI includes a free water fraction (FWF) parameter designed to capture the contamination of tissues by free water at the microstructural level.

In the present work, we assess the added value of NODDI compared to conventional DTI and anatomical MRI to detect changes at the presymptomatic phase of C9orf72 disease. We hypothesize that NDI and ODI offer higher sensitivity than FA, mean diffusivity (MD), axial diffusivity (AD) and radial diffusivity (RD) for detecting white matter abnormalities. 
Additionally, FWF is compared with volumetry and may provide complementary information for identifying gray matter changes.

\section{Material and Methods}

\section{Participants}

Eighty-six first-degree relatives of $C 9$ orf72 mutation carriers from 48 families were enrolled in an ongoing national multicentric study (PREV-DEMALS) between October 2015 and October 2017. This study was approved by the Comité de Prévention des Personnes Ile de France VI of the Hôpital Pitié-Salpêtrière, and written informed consent was obtained from all participants. At inclusion, asymptomatic status of participants was ascertained based on relative's interview, neurological examination, and the normality of behavioral scales and neuropsychological scores, taking into account age and educational level. In the present study, sixty-seven neurologically healthy participants, who underwent a single-shell diffusion weighted image (DWI) sequence, a multi-shell DWI sequence and a 3DT1 sequence, were included in the analyses. $C 9$ orf72 genetic status was determined by repeat-primed polymerase chain reaction on lymphocytes DNA. Thirty-eight $C 9+$ participants carried a pathogenic expansion $(>23$ GGGGCC repeats); twenty-nine control participants did not carry this expansion $\left(\mathrm{C}^{9-}\right)$. The study population characteristics are shown in Table 1. Demographic characteristics and clinical tests were compared between groups using the $\chi^{2}$ test for dichotomous and categorical variables or Mann-Whitney test for numerical variables. There was no statistical difference between $C 9+$ and $C 9$ - regarding age at inclusion $(P=0.18)$, gender $(P=0.65)$ and clinical scores (Mini-Mental State Examination, $P=0.83$; Mattis dementia rating scale, $P=0.37$; Frontal assessment battery, $P=0.40)$. 


\section{Table 1. Study Group Characteristics.}

Expected ages at onset of $C 9 \operatorname{orf} 72$ carriers were estimated by averaging the ages at onset of affected relatives, as in a previous study ${ }^{3}$.

Abbreviations: MMSE, Mini-Mental State Examination; MDRS, Mattis dementia rating scale; FAB, Frontal assessment battery; NA, not applicable. Results are given as mean (standard deviation) or number (percentage).

\begin{tabular}{|c|c|c|c|}
\hline \multirow[b]{2}{*}{ Characteristics } & \multicolumn{2}{|l|}{ Mean (SD) } & \multirow[b]{2}{*}{$P$ value } \\
\hline & $C 9-(\mathrm{n}=29)$ & $C 9+(\mathrm{n}=38)$ & \\
\hline \multicolumn{4}{|l|}{ Demographic characteristics } \\
\hline Age, y & $44.8(13.5)$ & $40.7(11.5)$ & 0.18 \\
\hline Female, No. (\%) & $17(59)$ & $19(50)$ & 0.65 \\
\hline Right laterality, No. (\%) & $24(83)$ & $33(87)$ & 0.91 \\
\hline Expected time to onset, years & NA & $18.6(11.4)$ & NA \\
\hline \multicolumn{4}{|l|}{ Family phenotype, No. $(\%)$} \\
\hline FTLD & $12(42)$ & $18(47)$ & \\
\hline ALS & $2(7)$ & $3(8)$ & \\
\hline FTLD and ALS & $14(48)$ & $17(45)$ & 0.91 \\
\hline Unavailable & $1(3)$ & 0 & \\
\hline \multicolumn{4}{|l|}{ Neuropsychological scores } \\
\hline MMSE score (maximum, 30) & $28.7(1.2)$ & $28.5(1.5)$ & 0.83 \\
\hline FAB (maximum, 18) & $16.9(1.3)$ & $17.1(1.2)$ & 0.40 \\
\hline MDRS score (maximum, 144) & $141.9(2.2)$ & $141.3(2.7)$ & 0.37 \\
\hline
\end{tabular}

\section{MRI acquisition}

All MRI acquisitions were performed on a 3-T MRI system (Siemens Prisma Syngo 3T) in a single center (Paris) belonging to the harmonized national network of the Centre d'Acquisition et de Traitement d'Images (http://cati-neuroimaging.com/). ${ }^{13}$ The Centre d'Acquisition et de Traitement d'Images performed onsite visits for the setup of imaging protocols and regular follow-up. Each participant underwent a 3DT1 sequence with the following parameters: voxel size $1.1 \times 1.1 \times 1.1 \mathrm{~mm}^{3} ; \mathrm{TE} / \mathrm{TR}=2.93 / 2200 \mathrm{~ms} ;$ Bandwidth $=240 \mathrm{~Hz}$. One single-shell DWI sequence with two repeats was acquired for DTI with the following parameters: voxel size $2.5 \times 2.5 \times 2.5 \mathrm{~mm}^{3} ;$ TE/TR $=90 / 7300 \mathrm{~ms} ;$ Bandwidth $=1580 \mathrm{~Hz} ; 64$ diffusion-weighted directions, $b$-value $=1000 \mathrm{~s} / \mathrm{mm}^{2}, 9 \mathrm{~T} 2$-weighted images $\left(b\right.$-value $=0 \mathrm{~s} / \mathrm{mm}^{2}$, referred to as $\mathrm{b} 0$ image). One field map image was acquired to estimate the susceptibility-induced off-resonance 
field. One three-shell DWI sequence with two repeats was acquired with reversed phase encoding directions for NODDI model: voxel size $2 \times 2 \times 2 \mathrm{~mm}^{3}$; TE/TR $=70 / 3000 \mathrm{~ms}$; Bandwidth $=2090 \mathrm{~Hz}$; 60, 32 and 9 diffusion-weighted directions at b-value $=2200,700$ and $300 \mathrm{~s} / \mathrm{mm}^{2}$ respectively; $13 \mathrm{~b} 0$ images. Of note, the DTI analysis was based on the single-shell data $\left(b=1000 \mathrm{~s} / \mathrm{mm}^{2}\right)$. Indeed, DTI model is known to be a poor representation of the DWI signals at the high b-value (> 2000). On the other hand, the low b-values (300 and 700) are acquired at prolonged TE designed to accommodate the high b-value. So these low b-values data are not representative of standard DTI data. Systematic quality checks of MRI results were performed by the Centre d'Acquisition et de Traitement d'Images as in previous work. ${ }^{6}$ All images were of satisfactory quality except for one T1 acquisition and one single-shell DWI acquisition from different individuals. These two images were excluded respectively from gray matter and white matter analyses.

\section{Anatomical MRI processing}

T1-weighted images were processed with the FreeSurfer image analysis suite (Version 5.3; https://surfer.nmr.mgh.harvard.edu/), including skull stripping, intensity normalization, cortical and subcortical segmentation, cortical surface reconstruction and parcellation of the cortex using the Desikan-Killiany atlas ${ }^{14}$. We studied gray matter volumes of 68 cortical regions of interest (ROIs) and 18 subcortical ROIs. The list of regions is provided in Supplementary material (supplementary-appendix e-1). All ROI volumes were normalized by total intracranial volume (TIV).

\section{DTI processing}

The single-shell DWI data were processed with the same approach as in previous work. ${ }^{6}$ To summarize, head motion was corrected by rigidly registering the raw DWI volumes to the 
average b0 image, and an affine registration was used to correct for eddy current-induced distortions. The field map image was used to estimate the susceptibility-induced off-resonance field. ${ }^{15} \mathrm{~A}$ single multiplicative bias field from the averaged b0 image was estimated and applied to the single-shell DWI data. ${ }^{16} \mathrm{FA}, \mathrm{MD}, \mathrm{RD}$ and $\mathrm{AD}$ were estimated using an iteratively reweighted linear least squares estimator. ${ }^{17}$ Each individual FA map was aligned onto the JHU white-matter tractography atlas template ${ }^{18}$ with a rigid plus deformable registration. ${ }^{19} \mathrm{MD}, \mathrm{AD}$ and RD maps were subsequently registered into the JHU atlas using the transformation field from the previous step. The regional mean values of FA, MD, AD and RD maps were extracted. The list of regions is provided in Supplementary material (supplementary-appendix e-2).

\section{NODDI processing}

From the pairs of images with reversed phase encoding directions, the susceptibility-induced off-resonance field was estimated using topup tool. ${ }^{20}$ Besides, eddy current-induced distortions and subject movements were corrected by simultaneously modelling the effects of diffusion eddy currents and movements on the image using eddy tool. ${ }^{21}$ The b-vector was subsequently corrected. The NODDI model was then fitted to the artifact-corrected data, generating NDI, ODI and FWF maps using NODDI Matlab toolbox (https://www.nitrc.org/projects/noddi toolbox). For white matter analysis, DTI model was applied to the middle-shell data $\left(b-v a l u e=700 \mathrm{~s} / \mathrm{mm}^{2}\right)$ to generate the FA map, in order to estimate the transformation field from the native diffusion space to the JHU space. Then NDI and ODI maps were registered into the JHU space using the transformation field obtained from the previous step. Finally, the regional mean values of NDI and ODI were calculated.

For gray matter analysis, the normalized skull-stripped T1 image in FreeSurfer conformed space was rigidly registered onto the first b0 image of the artifact-corrected multishell DWIs. Then the inverse transformation field was applied to register FWF map in the 
FreeSurfer conformed space with a linear interpolation. For subcortical ROI analysis, a 2-voxel morphological erosion operator was performed on each segmented subcortical ROI. ${ }^{22}$ For cortical ROI analysis, FWF signal was projected onto cortical middle surface. The projected FWF value was calculated with a weighted average of signals from the seven intermediate surfaces, which were expanded at different fraction of cortical thickness $(35 \%, 40 \%, 45 \%, 50 \%$, $55 \%, 60 \%$ and $65 \%$ ) from the white surface. Subsequently, the averaged middle surface was registered onto the FsAverage template in FreeSurfer conformed space using a surface-based registration method. ${ }^{23}$ The aim of the erosion and middle surface projection approach was to avoid the partial volume effect (i.e., elimination of free water contamination at the edges of ventricle and the borders of the brain parenchyma). Finally, the regional mean values of FWF were extracted for further cortical and subcortical analyses.

\section{Statistical analysis}

All statistical analyses were performed with R Version 3.4.0 (The R Foundation). Structural and microstructural differences between $C 9+$ and $C 9-$ participants were assessed using linear mixed-effects models. We used real age, gender and group (i.e., mutation status) as fixed effects and family membership as random effect measuring the difference between the average response in the family and in the whole population (supplementary-appendix e-3). Likelihood ratio test was used to test each effect and $P$ values were corrected by the Benjamini-Hochberg method with a significance level of $P<0.05$. Besides, the effect size of each ROI between $C 9+$ and $C 9$ - was also reported using Cohen's $f 2$. Effect sizes obtained for different models or metrics (e.g., NODDI vs DTI) were compared using permutation tests with 10000 iterations for significant ROIs.

\section{Results}

White matter analysis 
Figure 1 displays altered white matter tracks after correction for multiple comparisons. Compared with $C 9-, C 9+$ showed extensive alterations in white matter integrity (i.e., reduced NDI, elevated MD, $\mathrm{AD}$ and $\mathrm{RD}$ ), involving several fronto-temporal related tracts (i.e., bilateral inferior fronto-occipital fasciculus, bilateral inferior longitudinal fasciculus, right uncinate fasciculus, right anterior thalamic radiation, forceps minor) and both cortico-spinal tracts. 10 tracts were significantly altered in $C 9+$ with NDI, and only 5 tracts with DTI metrics (MD, AD or RD). Results before correction for multiple comparisons are shown in supplementary material (supplementary-figure e-1). Effect size results confirmed that NDI was more sensitive than DTI metrics: among the 11 tracts in which significant differences were detected in either NDI or DTI metrics (MD, AD or RD), 7 tracts had higher effect size with NDI than with DTI metrics (Figure 2). The effect size was significantly higher with NDI than with DTI for two of these tracts: left inferior fronto-occipital fasciculus $(P=0.009)$ and right uncinate fasciculus $(P$ $=0.008)$. None of the tracts had a significantly higher effect size with DTI than with NDI. Effect size results are shown in supplementary material (supplementary-table e-1). 
A FA

D RD

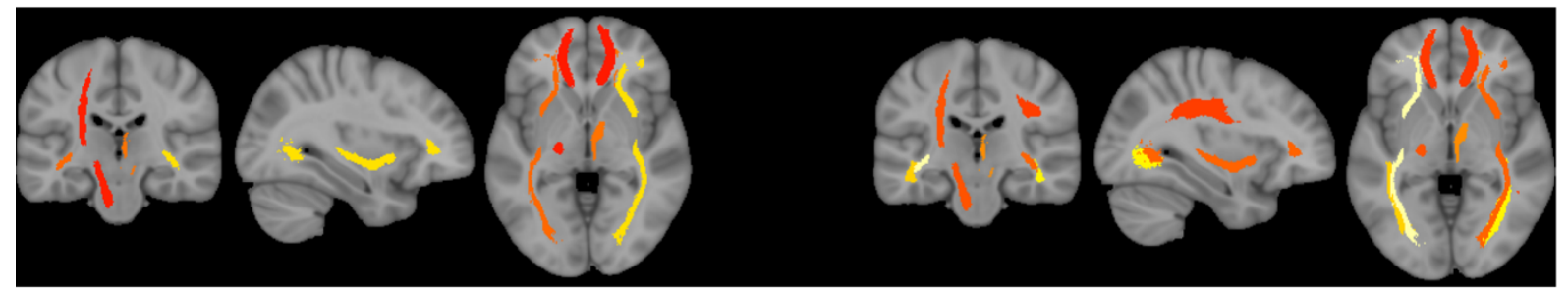

B MD

E NDI

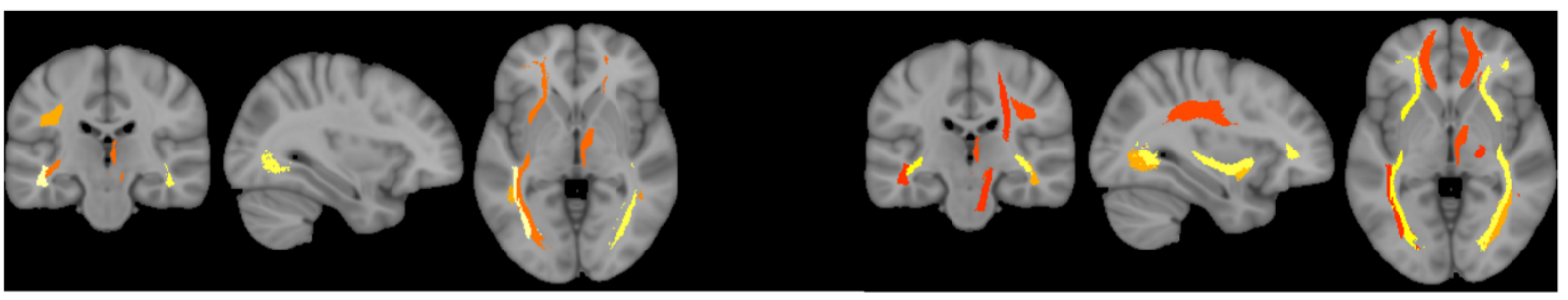

C $A D$

F ODI

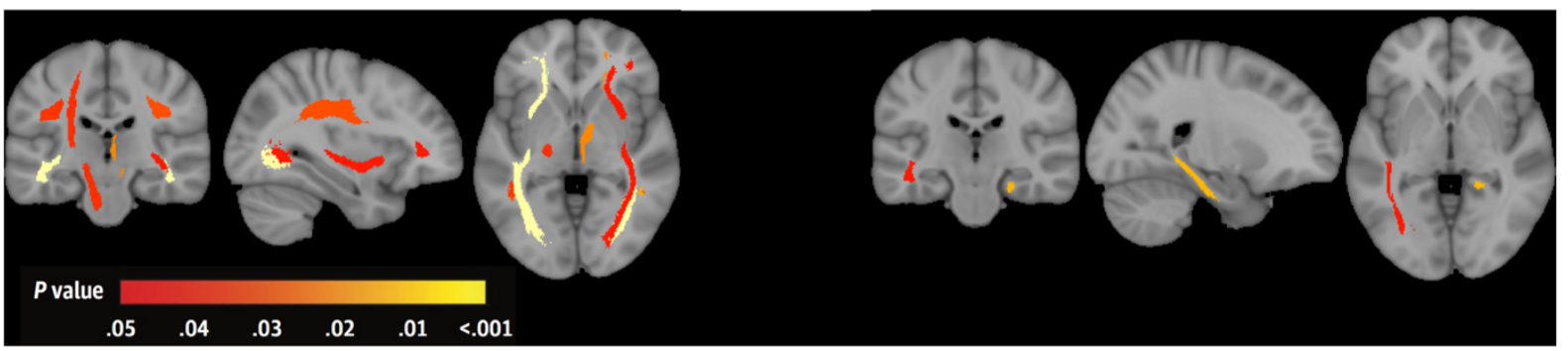

Figure 1. White Matter Alterations in C9orf72 Mutation Carriers. Color-coded

representation of $P$ values corresponding to the associations of $C 9$ orf 72 mutation with white matter integrity, both before (left) and after (right) correction for multiple comparisons.

Abbreviations: FA, fractional anisotropy; $\mathrm{MD}$, mean diffusivity; $\mathrm{AD}$, axial diffusivity; $\mathrm{RD}$, radial diffusivity; NDI, neurite density index; ODI, orientation dispersion index. 


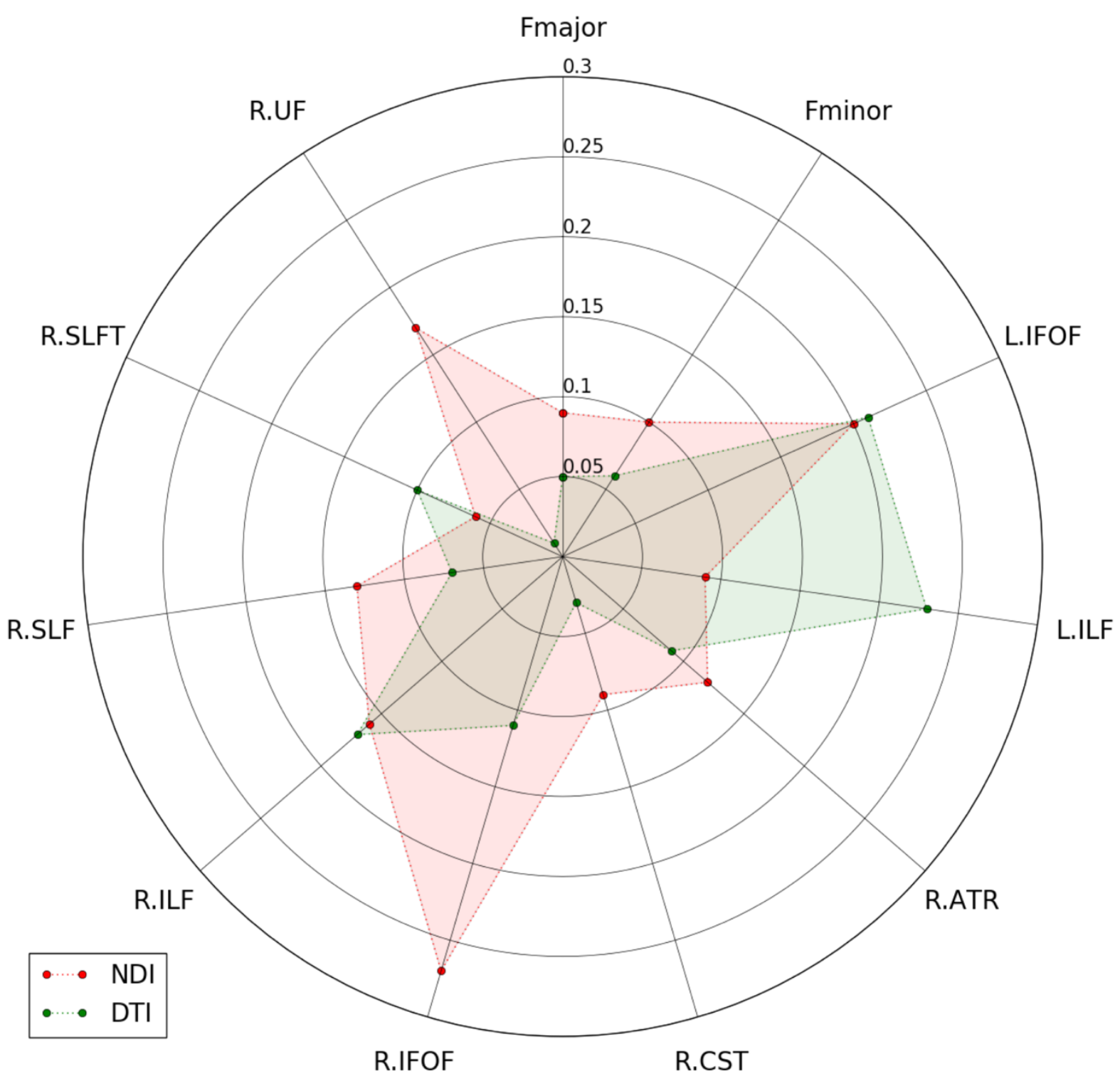

Figure 2. Effect Size of White Matter Alterations in C9orf72 Mutation Carriers. Effect size (Cohen's $f 2$ ) corresponding to the associations of $C 9$ orf72 mutation with white matter integrity. Only ROIs in which significance was detected in either NDI or DTI metrics are displayed. Abbreviations: ART, anterior thalamus radiation tract; CST, cortico-spinal tract; Fmajor, forceps major tract; Fminor, forceps minor tract; IFOF, inferior fronto-occipital fasciculus; IFOF, inferior fronto-occipital fasciculus; ILF, inferior longitudinal fasciculus; ILF, inferior longitudinal fasciculus; UF, uncinate fasciculus; SLFT, superior longitudinal 
fasciculus (temporal part); R., right; L., left; NDI, neurite density index; DTI, diffusion tensor imaging.

\section{Cortical gray matter analysis}

Figure 3 displays altered cortical regions after correction for multiple comparisons. Compared with $C 9-, C 9+$ showed reduced cortical gray matter volume and elevated FWF. 13 ROIs were significantly altered with FWF (4 frontal, 1 temporal, 4 parietal, 3 occipital and the left insula), and 11 ROIs with volumetry ( 2 frontal, 5 temporal and 4 parietal ROIs). Results before correction for multiple comparisons are shown in supplementary material (supplementaryfigure e-2). Figure 4 displays effect sizes for the 21 regions that were significantly altered according to either FWF or volumetry. Among the 21 regions, the left insula $(P=0.002)$, the lateral occipital cortex $(P=0.001)$ and the left pericalcarine cortex $(P=0.008)$ had a significantly higher effect size with FWF than with volumetry. Only the left temporal pole cortex showed significantly higher effect size with volumetry than with FWF $(P=0.02)$. Effect size results are shown in supplementary material (supplementary-table e-2).
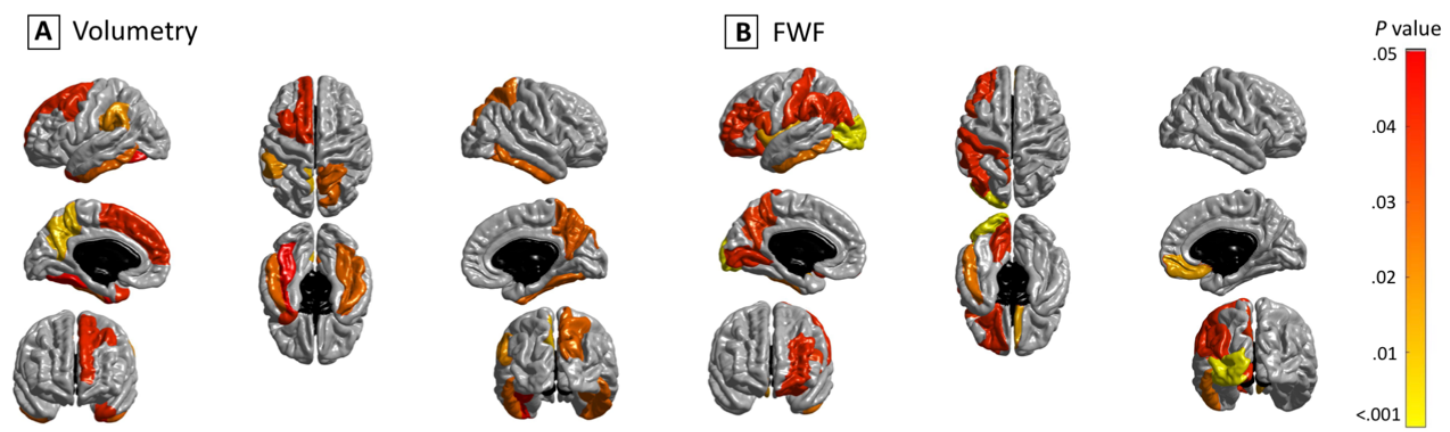

Figure 3. Cortical Alterations in C9orf 72 Mutation Carriers. Color-coded representation of $P$ values corresponding to the associations of $C 9 \operatorname{orf} 72$ mutation with the cortical ROI measures, both before (left) and after (right) correction for multiple comparisons. Abbreviations: FWF, free water fraction. 


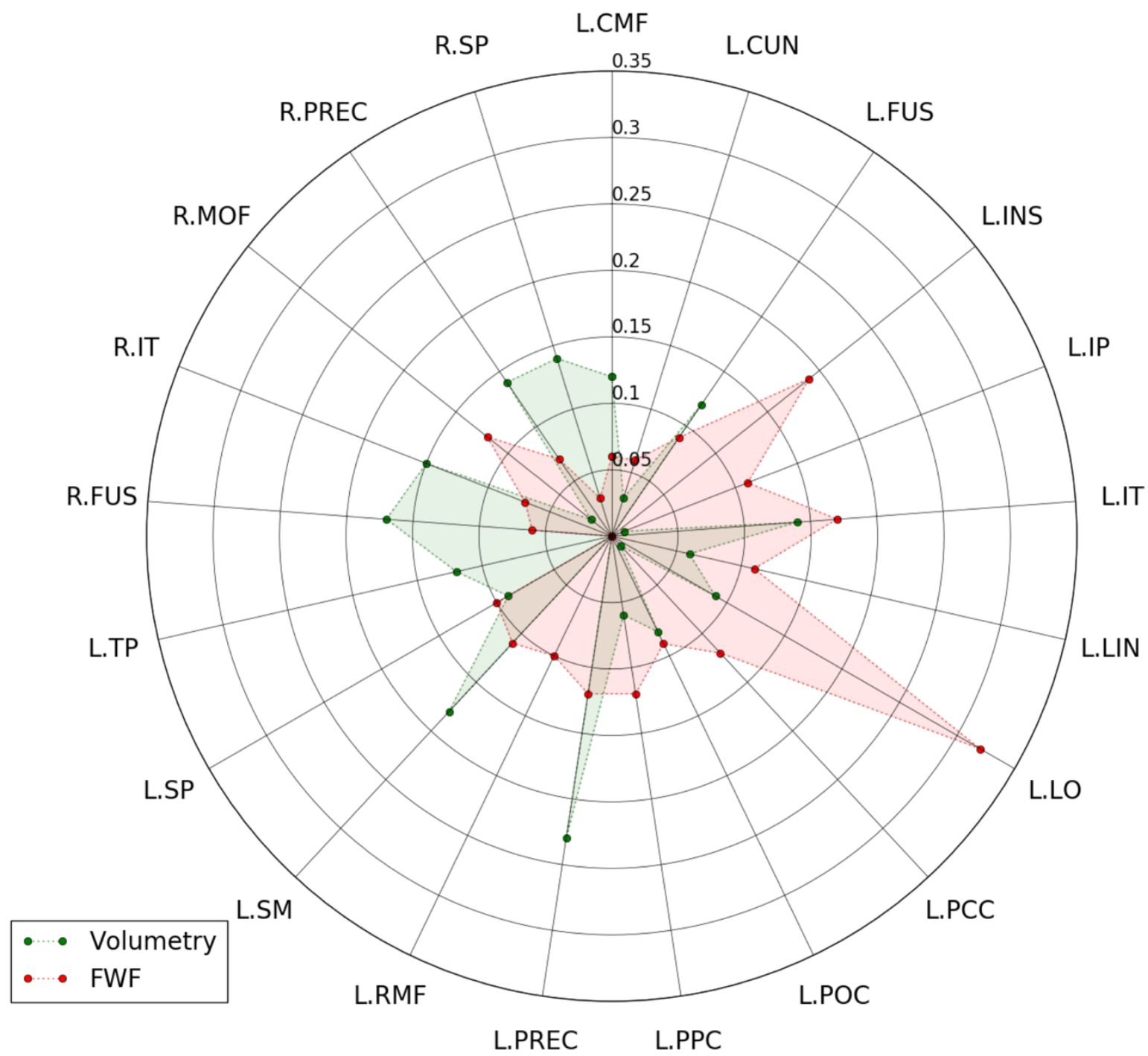

Figure 4. Effect Size of Cortical Alterations in C9orf 72 Mutation Carriers. Effect size (Cohen's $f 2$ ) corresponding to the associations of $C 9$ orf72 mutation with the cortical ROI measures. Only ROIs in which significance was detected in either volumetry or FWF are displayed. Abbreviations: CMF, caudal middle frontal cortex; CUN, cuneus cortex; FUS, fusiform; IP, inferior parietal cortex; IT, inferior temporal cortex; INS, insula; LO, lateral occipital cortex; LIN, lingual; PPC, pars opercularis cortex; PCC, pericalcarine; POC, postcentral cortex; PREC, precuneus; RMF, rostral middle frontal cortex; SP, superior 
parietal cortex; SM, supramarginal cortex; TP, temporal pole cortex; MOF, medial orbitofrontal cortex; R., right; L., left; FWF, free water fraction.

\section{Subcortical gray matter analysis}

Compared with $C 9-, C 9+$ showed subcortical volume reduction of the right thalamus after correction for multiple comparisons. FWF failed to detect any significant alterations (Figure 5). Results before correction for multiple comparisons are presented in supplementary material (supplementary-figure e-3). The right thalamus did not show statistically higher effect size with volumetry than with FWF $(P=0.062)$. Effect size results are shown in supplementary material (supplementary-table e-3).
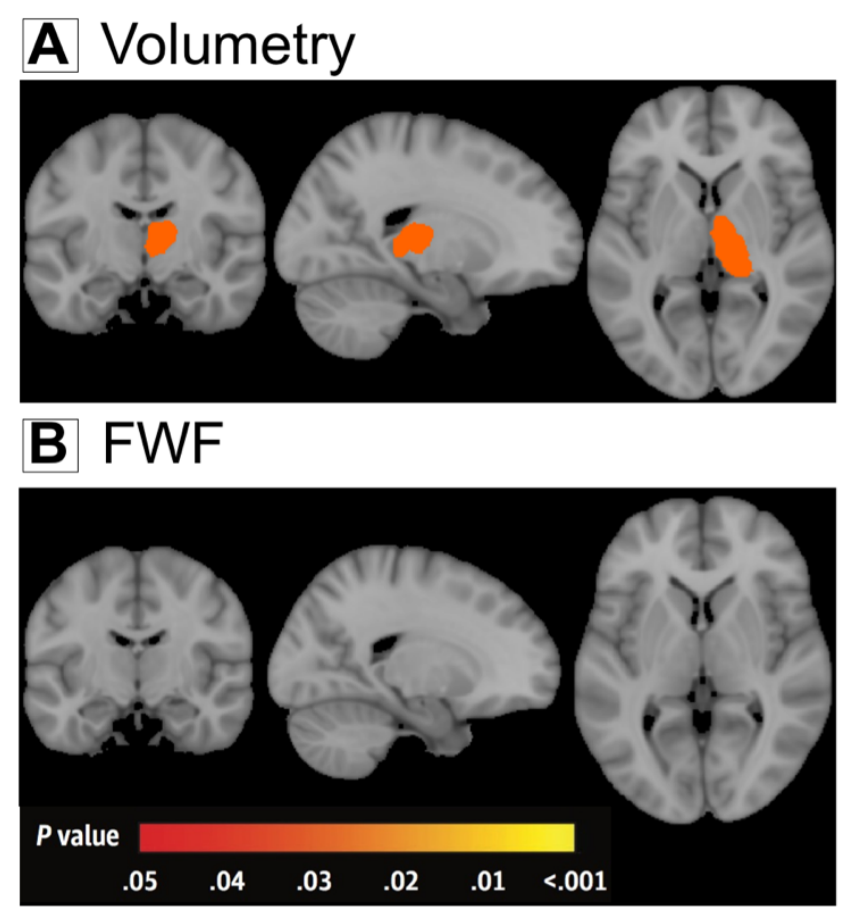

Figure 5. Subcortical Alterations in C9orf 72 Mutation Carriers. Color-coded representation of $P$ values corresponding to the associations of $C 9$ orf72 mutation with the subcortical ROI measures, both before (left) and after (right) correction for multiple comparisons. Abbreviations: FWF, free water fraction. 


\section{Discussion}

The current study, for the first time, compared NODDI to conventional DTI and anatomical MRI in a large cohort of presymptomatic $C 9$ orf 72 carriers. There are three key findings. First, we demonstrate that NODDI provides higher sensitivity than DTI for detecting white matter microstructural changes. Second, the greater tissue-specificity of NODDI suggests that the reduction of neurite density is a more likely cause of signal changes than the alterations of neurite orientation dispersion during the presymptomatic stage. Third, the pattern of FWF alterations slightly differs from that of gray matter volumetric atrophy, suggesting that both FWF and volumetry provide complimentary information on the integrity of cortical and subcortical structures.

When comparing the multi-shell DWI sequence with the standard single-shell DWI sequence, we hypothesized that NODDI could be more sensitive than conventional DTI for detecting white matter abnormalities during the presymptomatic stage. Previous works have shown that NODDI could be more sensitive than DTI for detecting white matter changes related to aging ${ }^{24}$ or young onset Alzheimer's disease. ${ }^{25}$ Here, for the first time, we show that NODDI outperformed DTI for identifying white matter abnormalities during the presymptomatic stage of a neurodegenerative disease. The spatial pattern of white matter changes is consistent with previous findings with conventional DTI ${ }^{5,6,8}$. Specifically, reduced NDI was mainly found in the cortico-spinal tract and frontal-temporal related tracts during the presymptomatic stage for C9orf72 carriers, which were preferentially involved in symptomatic mutation carriers who develop FTLD, ALS or both. On the other hand, to the best of our knowledge, there has been no prior study using NODDI in C9orf72 disease, neither at the presymptomatic nor at the symptomatic stage.

The interpretation of specificity for DTI metrics has been discussed, ${ }^{26,27}$ suggesting that $\mathrm{RD}$ and $\mathrm{AD}$ reflect respectively demyelination and axonal damage and both provide more 
specific information than FA. However, these interpretations were also argued in the literature. ${ }^{28}$ Compared with DTI, the more biophysically motivated NODDI model allows more direct analysis of independent microstructural effects: the loss of neurite density and the alteration of neurite orientation dispersion. This potential for greater tissue-specificity has motivated application of NODDI in a few neurodegenerative disease studies ${ }^{29,30,31,25,32}$. In young onset Alzheimer's disease, one study showed widespread NDI reduction and regional ODI reduction in the corpus callosum and internal capsule of patients. ${ }^{25}$ In Parkinson disease, another study observed reduced NDI in the substantia nigra and putamen of patients ${ }^{31}$. In the present study, we detected widespread NDI decrease in the white matter, but no alteration of ODI (Figure 1). This suggests that the reduction of neurite density, not the alterations of neurite orientation dispersion, is the predominant pathological process in white matter during the presymptomatic stage of $C 9$ orf72 carriers. Interestingly, similar reduction of neurite density within cortico-spinal tract have been found in ALS patients. ${ }^{33}$ Our observation suggests that the neurite loss is the main pathological process, but this needs further histological confirmation. We note however that Grussu et al have demonstrated, in the tissue specimen of multiple sclerosis, that ODI correlated well with histological measures of neurite orientation dispersion, and NDI with histological measures of neurite density. ${ }^{32}$

Mapping the free water in tissues is important in order to estimate the variations in extracellular volume (related to free water fraction) due to pathological processes. ${ }^{34} \mathrm{FWF}$ has been applied in several neurodegenerative diseases. Using a bi-tensor model, FWF has been demonstrated as an imaging biomarker of progression of Parkinson's disease in the posterior substantia nigra. ${ }^{35,36}$ Interestingly, a chronic treatment effect of rasagiline, an irreversible inhibitor of monoamine oxidase-B as a medical monotherapy, has been verified with FWF in basal ganglia in Parkinson's disease. ${ }^{37}$ These findings support the use of FWF as a promising biomarker in neurodegenerative diseases to evaluate the free water related alterations. In the 
present study, FWF detected free water alterations mainly in frontal and temporal lobes. This finding is consistent with previous studies using volumetric measure. . $^{3,4,5,6,7,8,9}$ The unique differences detected with FWF in left insula and left lateral occipital lobe were also reported in literatures using volumetric measure. ${ }^{3,4,5}$ These suggest that macroscopic brain atrophy may accompany free water alterations inside the cortex. Surprisingly, FWF failed to show changes in subcortical structures, such as the thalamic atrophy where a significant effect of $C 9$ orf 72 mutation was evidenced by volumetry. These findings suggest that distinct degenerative processes could occur in cortical and subcortical structures at the same time during the presymptomatic stage.

Reliability of an imaging technique is an important issue for its use in clinical trials. We do not have test-retest scans in our participants. However, the reliability of NODDI has been assessed in previous studies. ${ }^{38,39}$ NODDI metrics were shown to have excellent reproducibility with coefficients of variation below $5 \%$ in all measured regions of interest and even below $3 \%$ in the vast majority of regions ${ }^{39}$ Furthermore, the reproducibility of NODDI metrics was shown to be comparable to that of conventional DTI. ${ }^{38}$ This, together with its higher sensitivity to detect white matter alterations, supports the use of NODDI in clinical trials.

\section{Limitations}

Our study has the following limitations. First, the cross-model comparison between NODDI and DTI used two different DWI acquisitions, which were performed within one week sequentially for each participant. However, the single-shell and multi-shell DWI sequences were optimized for DTI and NODDI model, respectively. Thus, this systematic comparison helps clarify the added value of a longer but clinically feasible multi-shell diffusion sequence. Second, caution should be exercised in diffusion MRI-based cortical analysis. NODDI, by construction, accounts for partial volume effects from CSF contamination, thus minimizing the 
influence of atrophy on the NODDI metrics. Moreover, a recent paper ${ }^{40}$ has explicitly looked at the influence of cortical thickness on NODDI metrics, showing that the majority of changes in NODDI metrics persisted following adjustment for cortical thickness. Nevertheless, the cortex is a thin structure compared to the resolution of diffusion MRI and partial volume effect may impact on the computation of regional FWF measures. Although we implemented specific image processing procedures to mitigate partial volume effects, it is still possible that some partial volume effect remains, impacting on FWF estimates.

\section{Conclusion}

In conclusion, this study evaluated the added value of NODDI in detection of brain microstructural changes in presymptomatic $C 9$ orf 72 carriers. NODDI offers higher sensitivity compared to conventional DTI for detecting white matter integrity abnormalities. Moreover, it offers the potential to reveal a more specific substrate of white matter damage, suggesting here that it consists mainly of reduced neurite density. Our results encourage the use of a multi-shell DWI sequence in clinical studies and highlight the potential use of NODDI to track the presymptomatic stage of neurodegenerative diseases. 


\section{Study funding}

This study was funded by Assistance Publique - Hôpitaux de Paris (Clinical Research and Development Department), grant ANR/DGOS PRTS 2015-2019 PrevDemAls (to I.L.B.) and by "Investissements d'avenir" ANR-10-IAIHU-06 (Agence Nationale de la Recherche-10Investissements-Avenir-Institut-Hospitalo-Universitaire-06). EPSRC Grants EP/L022680/1 EP/M020533/1 and EP/N018702/1 support DCA and HZ's work on this topic. O.C. is supported by a "Contrat d'Interface Local” from Assistance Publique-Hôpitaux de Paris (APHP). The study was conducted with the support of the Centre d'Investigation Clinique (CIC 1422), and the Centre pour l'Acquisition et le Traitement des Images (CATI) platform, at IHUA-ICM, Paris, France. China Scholarship Council supports J.W's work on this topic.

This study has been registered on the website http://clinicaltrials.gov/ under number NCT02590276.

The funding sources had no role in design and conduct of the study; collection, management, analysis, and interpretation of the data; preparation, review, or approval of the manuscript; and decision to submit the manuscript for publication.

\section{Disclosure}

Competing financial interests related to the present article: none to disclose for all authors.

Competing financial interests unrelated to the present article: $\mathrm{OC}$ and SD are funded by European Union's Horizon 2020 research and innovation programme under grant agreement No 666992 (EuroPOND), and No. 720270 (HBP SGA1). SD is funded by European Research Council (ERC) under grant agreement No 678304. PC received consultant fees from Boehringer Ingelheim and funding for his institution from Cytokinetics. 


\section{References}

1. DeJesus-Hernandez M, Mackenzie IR, Boeve BF, et al. Expanded GGGGCC Hexanucleotide Repeat in Noncoding Region of C9ORF72 Causes Chromosome 9p-Linked FTD and ALS. Neuron. 2011;72(2):245-256. doi:10.1016/j.neuron.2011.09.011

2. Renton AE, Majounie E, Waite A, et al. A hexanucleotide repeat expansion in C9ORF72 is the cause of chromosome 9p21-linked ALS-FTD. Neuron. 2011;72(2):257-268. doi:10.1016/j.neuron.2011.09.010

3. Rohrer JD, Nicholas JM, Cash DM, et al. Presymptomatic cognitive and neuroanatomical changes in genetic frontotemporal dementia in the Genetic Frontotemporal dementia Initiative (GENFI) study: a cross-sectional analysis. Lancet Neurol. $2015 ; 14(3): 253-262$.

4. Walhout R, Schmidt R, Westeneng H-J, et al. Brain morphologic changes in asymptomatic C9orf72 repeat expansion carriers. Neurology. 2015;85(20):1780-1788.

5. Lee SE, Sias AC, Mandelli ML, et al. Network degeneration and dysfunction in presymptomatic C9ORF72 expansion carriers. NeuroImage Clin. 2016;14:286-297. doi:10.1016/j.nicl.2016.12.006

6. Bertrand A, Wen J, Rinaldi D, et al. Early Cognitive, Structural, and Microstructural Changes in Presymptomatic C9orf72 Carriers Younger Than 40 Years. JAMA Neurol. December 2017. doi:10.1001/jamaneurol.2017.4266

7. Cash DM, Bocchetta M, Thomas D, et al. Patterns of grey matter atrophy in genetic frontotemporal dementia: results from the GENFI study. Neurobiol Aging. October 2017. doi:10.1016/j.neurobiolaging.2017.10.008

8. Papma JM, Jiskoot LC, Panman JL, et al. Cognition and gray and white matter characteristics of presymptomatic C9orf72 repeat expansion. Neurology. 2017;89(12):12561264. doi:10.1212/WNL.0000000000004393 
9. Popuri K, Dowds E, Beg MF, et al. Gray matter changes in asymptomatic C9orf72 and GRN mutation carriers. NeuroImage Clin. doi:10.1016/j.nicl.2018.02.017

10. Alexander AL, Lee JE, Lazar M, Field AS. Diffusion Tensor Imaging of the Brain. Neurother J Am Soc Exp Neurother. 2007;4(3):316-329. doi:10.1016/j.nurt.2007.05.011

11. O’Donnell LJ, Westin C-F. An introduction to diffusion tensor image analysis. Neurosurg Clin N Am. 2011;22(2):185-viii. doi:10.1016/j.nec.2010.12.004

12. Zhang H, Schneider T, Wheeler-Kingshott CA, Alexander DC. NODDI: Practical in vivo neurite orientation dispersion and density imaging of the human brain. NeuroImage. 2012;61(4):1000-1016. doi:10.1016/j.neuroimage.2012.03.072

13. Operto G, Chupin M, Batrancourt B, et al. CATI: A Large Distributed Infrastructure for the Neuroimaging of Cohorts. Neuroinformatics. 2016;14(3):253-264. doi:10.1007/s12021-016-9295-8

14. Desikan RS, Ségonne F, Fischl B, et al. An automated labeling system for subdividing the human cerebral cortex on MRI scans into gyral based regions of interest. NeuroImage. 2006;31(3):968-980. doi:10.1016/j.neuroimage.2006.01.021

15. Jenkinson M, Smith S. A global optimisation method for robust affine registration of brain images. Med Image Anal. 2001;5(2):143-156.

16. Jeurissen B, Tournier J-D, Dhollander T, Connelly A, Sijbers J. Multi-tissue constrained spherical deconvolution for improved analysis of multi-shell diffusion MRI data. NeuroImage. 2014;103:411-426. doi:10.1016/j.neuroimage.2014.07.061

17. Tournier J-D, Calamante F, Connelly A. MRtrix: Diffusion tractography in crossing fiber regions. Int J Imaging Syst Technol. 2012;22(1):53-66. doi:10.1002/ima.22005 18. Hua K, Zhang J, Wakana S, et al. Tract Probability Maps in Stereotaxic Spaces: Analyses of White Matter Anatomy and Tract-Specific Quantification. NeuroImage. 2008;39(1):336-347. doi:10.1016/j.neuroimage.2007.07.053 
19. Avants BB, Epstein CL, Grossman M, Gee JC. Symmetric Diffeomorphic Image Registration with Cross-Correlation: Evaluating Automated Labeling of Elderly and Neurodegenerative Brain. Med Image Anal. 2008;12(1):26-41. doi:10.1016/j.media.2007.06.004

20. Andersson JLR, Skare S, Ashburner J. How to correct susceptibility distortions in spin-echo echo-planar images: application to diffusion tensor imaging. NeuroImage. 2003;20(2):870-888. doi:10.1016/S1053-8119(03)00336-7

21. Andersson JLR, Sotiropoulos SN. An integrated approach to correction for offresonance effects and subject movement in diffusion MR imaging. Neuroimage. 2016;125:1063-1078. doi:10.1016/j.neuroimage.2015.10.019

22. Pfefferbaum A, Adalsteinsson E, Rohlfing T, Sullivan EV. Diffusion tensor imaging of deep gray matter brain structures: Effects of age and iron concentration. Neurobiol Aging. 2010;31(3):482-493. doi:10.1016/j.neurobiolaging.2008.04.013

23. Fischl B, Sereno MI, Tootell RBH, Dale AM. High-resolution intersubject averaging and a coordinate system for the cortical surface. Hum Brain Mapp. 1999;8(4):272-284. doi:10.1002/(SICI)1097-0193(1999)8:4<272::AID-HBM10>3.0.CO;2-4

24. Kodiweera C, Alexander AL, Harezlak J, McAllister TW, Wu Y-C. Age effects and sex differences in human brain white matter of young to middle-aged adults: A DTI, NODDI, and q-space study. NeuroImage. 2016;128:180-192. doi:10.1016/j.neuroimage.2015.12.033 25. Slattery CF, Zhang J, Paterson RW, et al. ApoE influences regional white-matter axonal density loss in Alzheimer's disease. Neurobiol Aging. 2017;57:8-17. doi:10.1016/j.neurobiolaging.2017.04.021

26. Song S-K, Sun S-W, Ramsbottom MJ, Chang C, Russell J, Cross AH. Dysmyelination revealed through MRI as increased radial (but unchanged axial) diffusion of water. NeuroImage. 2002;17(3):1429-1436. 
27. DeBoy CA, Zhang J, Dike S, et al. High resolution diffusion tensor imaging of axonal damage in focal inflammatory and demyelinating lesions in rat spinal cord. Brain J Neurol. 2007;130(Pt 8):2199-2210. doi:10.1093/brain/awm122

28. Wheeler-Kingshott CAM, Cercignani M. About "axial" and "radial" diffusivities. Magn Reson Med. 2009;61(5):1255-1260. doi:10.1002/mrm.21965

29. Song Y-K, Li X-B, Huang X-L, et al. A study of neurite orientation dispersion and density imaging in wilson's disease. J Magn Reson Imaging JMRI. December 2017. doi:10.1002/jmri.25930

30. Schneider T, Brownlee W, Zhang H, Ciccarelli O, Miller DH, Wheeler-Kingshott CG. Sensitivity of multi-shell NODDI to multiple sclerosis white matter changes: a pilot study. Funct Neurol. 2017;32(2):97-101. doi:10.11138/FNeur/2017.32.2.097

31. Kamagata K, Hatano T, Okuzumi A, et al. Neurite orientation dispersion and density imaging in the substantia nigra in idiopathic Parkinson disease. Eur Radiol. 2016;26(8):25672577. doi:10.1007/s00330-015-4066-8

32. Grussu F, Schneider T, Tur C, et al. Neurite dispersion: a new marker of multiple sclerosis spinal cord pathology? Ann Clin Transl Neurol. 2017;4(9):663-679. doi:10.1002/acn3.445

33. Broad R, Gabel M, Dowell N, et al. Neurite Orientation Dispersion and Density Imaging (NODDI) highlights axonal degeneration of the motor tracts as the core feature underlying Amyotrophic Lateral Sclerosis. (S53.008). Neurology. 2017;88(16 Supplement):S53.008.

34. Pasternak O, Sochen N, Gur Y, Intrator N, Assaf Y. Free water elimination and mapping from diffusion MRI. Magn Reson Med. 2009;62(3):717-730.

doi:10.1002/mrm.22055

35. Planetta PJ, Ofori E, Pasternak O, et al. Free-water imaging in Parkinson's disease and 
atypical parkinsonism. Brain J Neurol. 2016;139(Pt 2):495-508. doi:10.1093/brain/awv361 36. Burciu RG, Ofori E, Archer DB, et al. Progression marker of Parkinson's disease: a 4year multi-site imaging study. Brain J Neurol. 2017;140(8):2183-2192.

doi:10.1093/brain/awx146

37. Burciu RG, Ofori E, Shukla P, et al. Free-water and BOLD imaging changes in Parkinson's disease patients chronically treated with a MAO-B inhibitor. Hum Brain Mapp. 2016;37(8):2894-2903. doi:10.1002/hbm.23213

38. Tariq M, Schneider T, Alexander DC, Wheeler-Kingshott CAM, Zhang H. Scanrescan reproducibility of neurite microstructure estimates using NODDI. :8.

39. Chang YS, Owen JP, Pojman NJ, et al. White Matter Changes of Neurite Density and Fiber Orientation Dispersion during Human Brain Maturation. Gong G, ed. PLOS ONE. 2015;10(6):e0123656. doi:10.1371/journal.pone.0123656

40. Parker TD, Slattery CF, Zhang J, et al. Cortical microstructure in young onset Alzheimer's disease using neurite orientation dispersion and density imaging. Hum Brain Mapp. doi:10.1002/hbm.24056 


\section{Supplementary material}

Appendix e-1: Regions for gray matter analyses.

Appendix e-2: Regions for white matter analyses.

Appendix e-3: Mixed effects model.

Figure e-1: Color-coded representation of $P$ values corresponding to the associations of $C 9$ orf72 mutation with white matter integrity before correction for multiple comparisons.

Figure e-2: Color-coded representation of $P$ values corresponding to the associations of $C 9$ orf 72 mutation with the cortical ROI measures before correction for multiple comparisons.

Figure e-3: Color-coded representation of $P$ values corresponding to the associations of C9orf72 mutation with the subcortical ROI measures before correction for multiple comparisons.

Table e-1: Effect sizes for DTI metrics and for NDI in white matter tracts.

Table e-2: Effect sizes for FWF and gray matter volume in cortical regions.

Table e-3: Effect sizes for FWF and gray matter volume in subcortical regions. 


\section{Appendix e-1. Regions for gray matter analyses.}

We studied the following 68 cortical regions obtained from the Desikan-Killiany atlas:

Frontal lobe: left frontal pole, left medial orbitofrontal, left lateral orbitofrontal, left pars orbitalis, left pars triangularis, left pars opercularis, left rostral middle frontal, left caudal, middle frontal, left superior frontal, left precentral, right frontal pole, right medial orbitofrontal, right lateral orbitofrontal, right pars orbitalis, right pars triangularis, right pars opercularis, right rostral middle frontal, right caudal middle frontal, right superior frontal, right precentral.

Temporal lobe: left temporal pole, left banks sts, left transverse temporal, left superior temporal, left middle temporal, left inferior temporal, left fusiform, left entorhinal, left parahippocampal, right temporal pole, right banks sts , right transverse temporal, right superior temporal, right middle temporal, right inferior temporal, right fusiform, right entorhinal, right parahippocampal.

Parietal lobe: left postcentral, left superior parietal, left inferior parietal, left precuneus, left supramarginal, left paracentral, right postcentral, right superior parietal, right inferior parietal, right precuneus, right supramarginal, right paracentral.

Occipital lobe: left lingual, left lateral occipital, left cuneus, left pericalcarine, right lingual, right lateral occipital, right cuneus, right pericalcarine.

Cingulate gyrus: left rostral anterior cingulate, left caudal anterior cingulate, left isthmus cingulate, left posterior cingulate, right rostral anterior cingulate, right caudal anterior cingulate, right isthmus cingulate, right posterior cingulate.

Insula: left insula, right insula.

The following 18 subcortical regions were included for gray matter volume analyses:

left cerebellum cortex, right cerebellum cortex, left ventral diencephalon, right ventral diencephalon, left putamen, right putamen, left pallidum, right pallidum, left caudate, right caudate, left accumbens area, right accumbens area, left amygdala, right amygdala, left thalamus proper, right thalamus proper, left hippocampus, right hippocampus.

The following 12 subcortical regions were included for gray matter FWF analyses. The other 6 ROIs were excluded because, after the 2-voxel erosion procedure, they were too small to produce reliable regional estimates.

left putamen, right putamen, left pallidum, right pallidum, left caudate, right caudate, left amygdala, right amygdala, left thalamus proper, right thalamus proper, left hippocampus, right hippocampus.

\section{Appendix e-2: Regions for white matter analyses.}

We studied the following 20 tracts based on JHU atlas for white matter analyses:

left anterior thalamic radiation, right anterior thalamic radiation, left corticospinal tract, right corticospinal tract, left cingulum cingulate gyrus, right cingulum cingulate gyrus, left cingulum hippocampus, right cingulum hippocampus, forceps major, forceps major, left inferior frontooccipital fasciculus, right inferior fronto-occipital fasciculus, left inferior longitudinal fasciculus, right inferior longitudinal fasciculus, left superior longitudinal fasciculus, right superior longitudinal fasciculus, right uncinate fasciculus, left uncinate fasciculus, left superior longitudinal fasciculus temporal, right superior longitudinal fasciculus temporal. 


\section{Appendix e-3. Mixed effects model.}

Group differences between carriers and non-carriers of the C9orf72 mutation were assessed using linear mixed-effects models. We used age, gender and group (i.e., mutation status) as fixed effects, and family membership as random effect, with the following model:

$$
Y_{i k}^{(j)}=\mu+\beta \times \text { Gender }_{i}+\lambda \times \text { Age }_{i}+\eta \times \operatorname{Group}_{i}+\mathrm{U}_{k}+E_{i k}^{(j)}
$$

where $\mathrm{Y}_{\mathrm{ik}}{ }^{(\mathrm{j})}$ is the response of the $\mathrm{j}^{\text {th }}$ region of interest (ROI) for the $\mathrm{i}^{\text {th }}$ subject and the $\mathrm{k}^{\text {th }}$

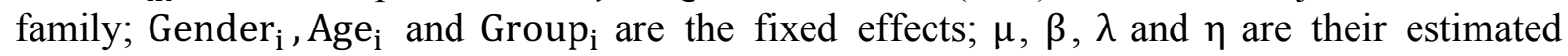
parameters; $U_{k}$ is the random effect measuring the difference between the average response in the family and in the whole population; $\mathrm{E}_{\mathrm{ik}}{ }^{(\mathrm{j})}$ is the random error.

Figure e-1. Color-coded representation of $P$ values corresponding to the associations of $C 90 r f 72$ mutation with white matter integrity before correction for multiple comparisons.

Abbreviations: FA, fractional anisotropy; MD, mean diffusivity; AD, axial diffusivity; RD, radial diffusivity; NDI, neurite density index; ODI, orientation dispersion index.
A FA
D RD

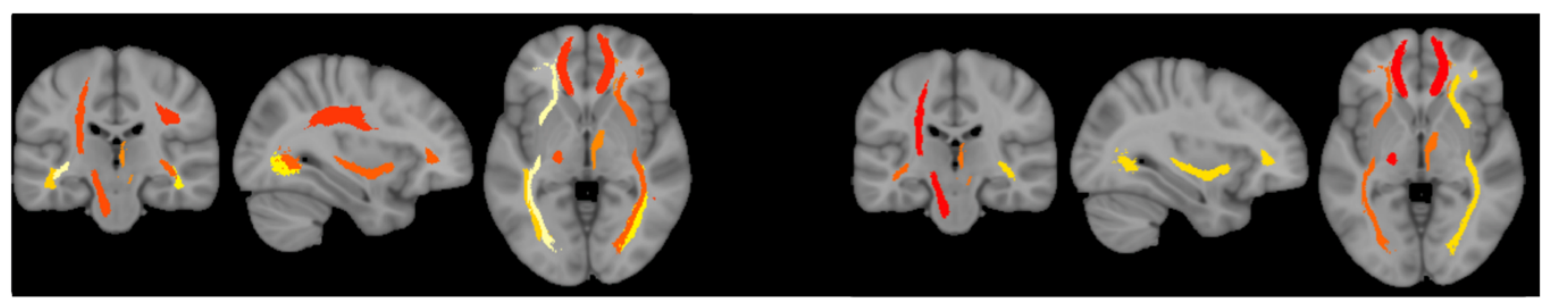

B $\mathrm{MD}$

E NDI

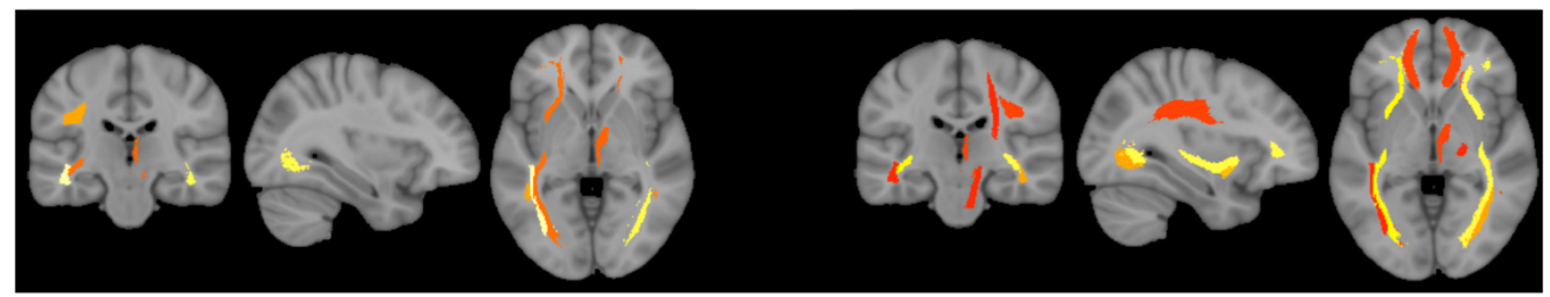

C $A D$

F ODI

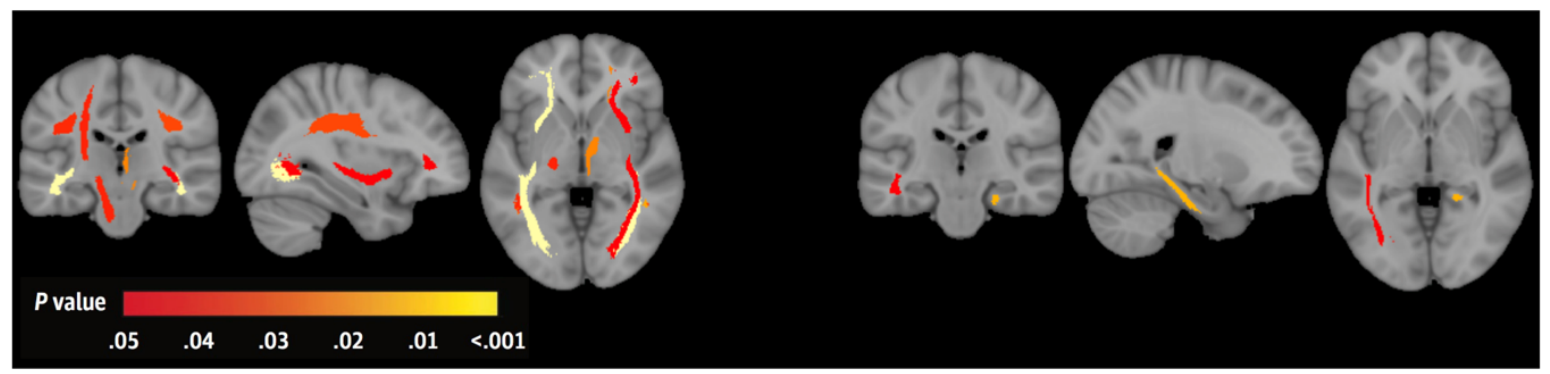


Table e-1. Effect sizes for DTI metrics and for NDI in white matter tracts.

The effect sizes for both NDI and DTI are shown below. For DTI, only the largest effect size among the different DTI metrics is shown. For regions for which a significant difference between $\mathrm{C} 9+$ and $\mathrm{C} 9$ - was detected for any of the metrics, we compared the effect size of NODDI to that of DTI using a permutation test procedures with 10000 iterations. The table presents the $P$ values corresponding to these permutation tests (with a significance level of $P<$ $0.05)$.

Abbreviations: ART, anterior thalamus radiation tract; CST, cortico-spinal tract; CCG, Cingulum (cingulate gyrus) tract; $\mathrm{CH}$, Cingulum (hippocampus) tract; Fmajor, forceps major tract; Fminor, forceps minor tract; IFOF, inferior fronto-occipital fasciculus; ILF, inferior longitudinal fasciculus; SLF, superior longitudinal fasciculus; UF, uncinate fasciculus; SLFT, superior longitudinal fasciculus (temporal part); R., right; L., left; NDI, neurite density index; DTI, diffusion tensor imaging; NA, not applicable.

\begin{tabular}{|c|c|c|c|c|c|}
\hline ROI & $\begin{array}{c}\text { Effect size } \\
\text { (NDI/DTI) }\end{array}$ & $\boldsymbol{P}$ value & ROI & $\begin{array}{c}\text { Effect size } \\
\text { (NDI/DTI) }\end{array}$ & $\boldsymbol{P}$ value \\
\hline L.ART & $0.06 / 0.04$ & NA & L.IFOF & $0.2 / 0.21$ & 0.773 \\
R.ATR & $0.12 / 0.09$ & 0.365 & R.IFOF & $0.27 / 0.11$ & $0.009^{*}$ \\
L.CST & $0.05 / 0.07$ & NA & L.ILF & $0.09 / 0.23$ & 0.051 \\
R.CST & $0.09 / 0.03$ & 0.154 & R.ILF & $0.16 / 0.17$ & 0.719 \\
L.CCG & $0.03 / 0.04$ & NA & L.SLF & $0.08 / 0.06$ & NA \\
R.CCG & $0.02 / 0.02$ & NA & R.SLF & $0.13 / 0.07$ & 0.139 \\
L.CH & $0.01 / 0.05$ & NA & L.UF & $0.03 / 0.02$ & NA \\
R.CH & $0.07 / 0.01$ & NA & R.UF & $0.17 / 0.01$ & $0.008^{*}$ \\
Fmajor & $0.09 / 0.05$ & 0.207 & L.SLFT & $0.06 / 0.05$ & NA \\
Fminor & $0.1 / 0.06$ & 0.307 & R.SLFT & $0.06 / 0.1$ & 0.258 \\
\hline
\end{tabular}

Figure e-2. Color-coded representation of $P$ values corresponding to the associations of $C 90 r f 72$ mutation with the cortical ROI measures before correction for multiple comparisons.

Abbreviations: FWF, free water fraction.

A Volumetry

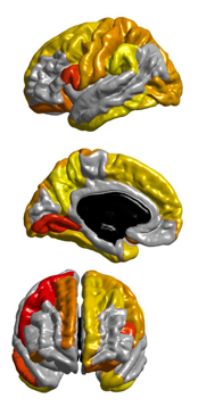

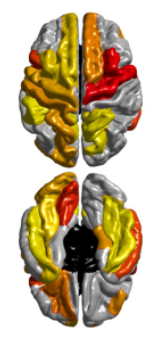
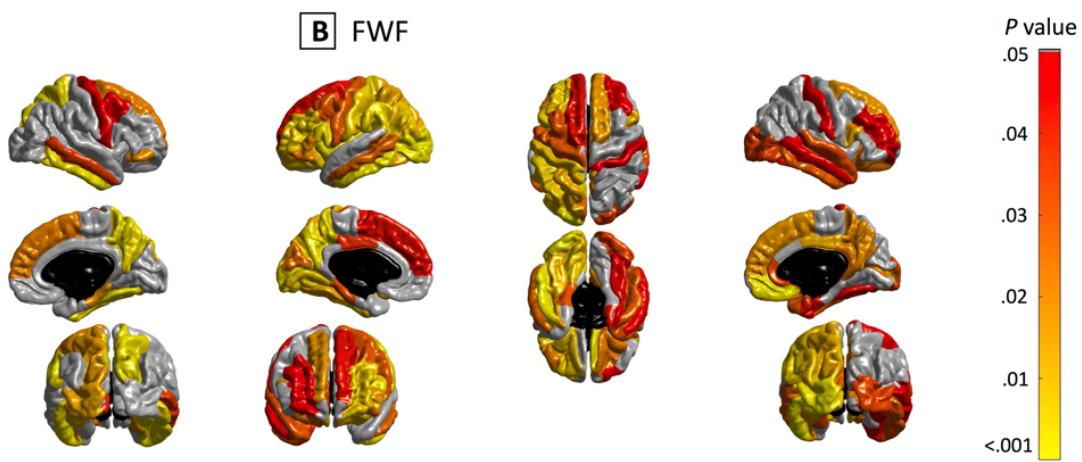


\section{Table e-2: Effect sizes for FWF metrics and gray matter volume in cortical regions.}

The effect sizes for both FWF and gray matter volume in cortical regions are shown below. For regions for which a significant difference between $\mathrm{C} 9+$ and $\mathrm{C} 9-$ was detected for any of the metrics, we compared the effect size of FWF to that of volumetry using a permutation test procedures with 10000 iterations. The table presents the $P$ values corresponding to these permutation tests (with a significance level of $P<0.05$ ).

Abbreviations: CMF, caudal middle frontal cortex; CUN, cuneus cortex; FUS, fusiform; IP, inferior parietal cortex; IT, inferior temporal cortex; INS, insula; LO, lateral occipital cortex; LIN, lingual; PPC, pars opercularis cortex; PCC, pericalcarine; POC, postcentral cortex; PREC, precuneus; RMF, rostral middle frontal cortex; SP, superior parietal cortex; SM, supramarginal cortex; TP, temporal pole cortex; MOF, medial orbitofrontal cortex; BK, bankssts cortex; CAC, caudal anterior cingulate cortex; ENT, entorhinal cortex; FP, frontal pole cortex; IC, isthmus cingulate cortex; LOF, lateral orbitofrontal cortex; MT, middle temporal cortex; PAC, paracentral cortex; PHC, para hippocampal cortex; PB, pars orbitalis cortex; PTR, pars triangularis cortex; PCG, posterior cingulate cortex; PRC, precentral cortex; RAC, rostral anterior cingulate cortex; SF, superior frontal cortex; ST, superior temporal cortex; TT, transverse temporal cortex; R., right; L., left; FWF, free water fraction; NA, not applicable.

\begin{tabular}{|c|c|c|c|c|c|}
\hline ROI & $\begin{array}{c}\text { Effect size } \\
\text { (FWF/Volumetry) }\end{array}$ & $\boldsymbol{P}$ value & ROI & $\begin{array}{c}\text { Effect size } \\
\text { (FWF/Volumetry) }\end{array}$ & $\boldsymbol{P}$ value \\
\hline L.CMF & $0.06 / 0.12$ & 0.734 & L.PREC & $0.12 / 0.23$ & 0.466 \\
L.CUN & $0.06 / 0.03$ & 0.653 & L.RMF & $0.1 / 0$ & 0.052 \\
L.FUS & $0.09 / 0.12$ & 0.305 & L.SP & $0.1 / 0.09$ & 0.712 \\
L.IP & $0.11 / 0.01$ & 0.072 & L.SM & $0.11 / 0.18$ & 0.639 \\
L.IT & $0.17 / 0.14$ & 0.259 & L.TP & $0 / 0.12$ & $0.025^{*}$ \\
L.INS & $0.19 / 0$ & $0.002^{*}$ & R.FUS & $0.06 / 0.17$ & 0.096 \\
L.LO & $0.32 / 0.09$ & $0.001^{*}$ & R.IT & $0.07 / 0.15$ & 0.406 \\
L.LIN & $0.11 / 0.06$ & 0.092 & R.MOF & $0.12 / 0.02$ & 0.051 \\
L.PPC & $0.12 / 0.06$ & 0.201 & R.PREC & $0.07 / 0.14$ & 0.483 \\
L.PCC & $0.12 / 0.01$ & $0.008 *$ & R.SP & $0.03 / 0.14$ & 0.140 \\
L.POC & $0.09 / 0.08$ & 0.558 & L.PB & $0.08 / 0$ & NA \\
R.CMF & $0.04 / 0.06$ & NA & L.PTR & $0.08 / 0.01$ & NA \\
L.BK & $0.08 / 0.01$ & NA & L.PCG & $0.06 / 0$ & NA \\
L.CAC & $0.04 / 0.01$ & NA & L.PRC & $0.07 / 0.09$ & NA \\
L.ENT & $0.02 / 0.01$ & NA & L.RAC & $0 / 0.01$ & NA \\
L.FP & $0.02 / 0.1$ & NA & L.SF & $0.06 / 0.11$ & NA \\
L.IC & $0.01 / 0.02$ & NA & L.ST & $0.01 / 0.04$ & NA \\
L.LOF & $0.11 / 0.06$ & NA & L.TT & $0.01 / 0.04$ & NA \\
L.MOF & $0.01 / 0.01$ & NA & R.BK & $0.05 / 0.01$ & NA \\
L.MT & $0.08 / 0.01$ & NA & R.CAC & $0.04 / 0.03$ & NA \\
L.PAC & $0.02 / 0.02$ & NA & R.CUN & $0 / 0.04$ & NA \\
L.PHC & $0.07 / 0$ & NA & R.ENT & $0.06 / 0$ & NA \\
R.FP & $0.04 / 0$ & NA & R.IP & $0.01 / 0.01$ & NA \\
R.INS & $0.05 / 0.02$ & NA & R.IC & $0.04 / 0$ & NA \\
R.LOF & $0.08 / 0.01$ & NA & R.LO & $0.06 / 0.04$ & NA \\
R.LIN & $0.04 / 0.02$ & NA & R.MT & $0.06 / 0.07$ & NA \\
R.PAC & $0.05 / 0.03$ & NA & R.PRC & $0.03 / 0.06$ & NA \\
R.PHC & $0.03 / 0.09$ & NA & R.RAC & $0.08 / 0.02$ & NA \\
R.PPC & $0.09 / 0.03$ & NA & R.RMF & $0.06 / 0.02$ & NA
\end{tabular}




\begin{tabular}{|c|c|c|c|c|c|} 
R.PB & $0.04 / 0.08$ & NA & R.SF & $0.09 / 0.08$ & NA \\
R.PTR & $0.04 / 0.03$ & NA & R.ST & $0.07 / 0.04$ & NA \\
R.PCC & $0.09 / 0.01$ & NA & R.SM & $0.03 / 0.05$ & NA \\
R.POC & $0.05 / 0.05$ & NA & R.TP & $0.04 / 0.06$ & NA \\
R.PCG & $0.09 / 0$ & NA & R.TT & $0 / 0.01$ & NA \\
\hline
\end{tabular}

Figure e-3. Color-coded representation of $P$ values corresponding to the associations of $C 9$ orf 72 mutation with the subcortical ROI measures before correction for multiple comparisons.

Abbreviations: FWF, free water fraction.
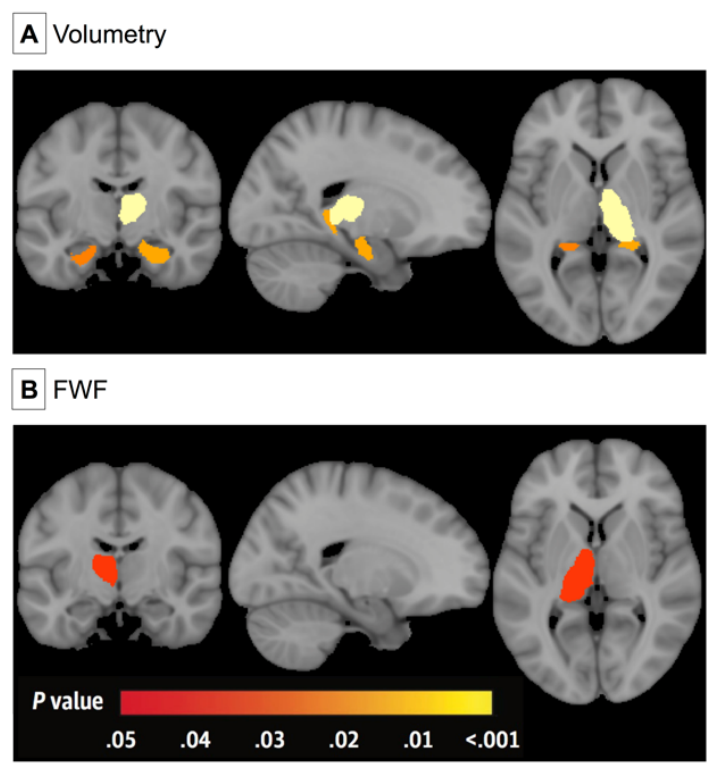

Table e-3. Effect sizes for FWF metrics and gray matter volume in subcortical regions.

The effect sizes for both FWF and gray matter volume in subcortical regions are shown below. For regions for which a significant difference between $\mathrm{C} 9+$ and $\mathrm{C} 9$ - was detected for any of the metrics, we compared the effect size of FWF to that of volumetry using a permutation test procedures with 10000 iterations. The table presents the $P$ values corresponding to these permutation tests (with a significance level of $P<0.05$ ). For FWF effect size analysis, 6 ROIs were excluded because, after the 2-voxel erosion procedure, they were too small to produce reliable regional estimates.

Abbreviations: $\mathrm{CB}$, cerebellum cortex; VD, ventral diencephalon; PUT, putamen; PAL, pallidum; CAU, caudate; ACC, accumbens area; THA, thalamus; HIP, hippocampus; FWF, free water fraction; NA, not applicable.

\begin{tabular}{|c|c|c|c|c|c|}
\hline ROI & $\begin{array}{c}\text { Effect size } \\
\text { (FWF/Volumetry) }\end{array}$ & $\boldsymbol{P}$ value & ROI & $\begin{array}{c}\text { Effect size } \\
(\text { FWF/Volumetry })\end{array}$ & $\boldsymbol{P}$ value \\
\hline L. CB & NA/0 & NA & R.CAU & $0.01 / 0$ & NA \\
R.CB & NA/0 & NA & L.ACC & NA/0 & NA
\end{tabular}




\begin{tabular}{|l|c|c|c|c|c|} 
L.VD & NA/0 & NA & R.ACC & NA/0 & NA \\
R.VD & NA/0 & NA & L.AMY & $0 / 0.03$ & NA \\
L.PUT & $0.05 / 0.03$ & NA & R.AMY & $0 / 0.04$ & NA \\
R.PUT & $0.03 / 0.05$ & NA & L.THA & $0.06 / 0.05$ & NA \\
L.PAL & $0 / 0.02$ & NA & R.THA & $0.03 / 0.16$ & 0.062 \\
R.PAL & $0.09 / 0.02$ & NA & L.HIP & $0 / 0.10$ & NA \\
L.CAU & $0.02 / 0.00$ & NA & R.HIP & $0 / 0.11$ & NA \\
\hline
\end{tabular}

